\title{
Oridonin induces apoptosis and reverses drug resistance in cisplatin resistant human gastric cancer cells
}

\author{
ZHONGWEI HE ${ }^{1,2^{*}}$, XIANGLING XIAO ${ }^{1,2^{*}}$, SHAN LI $^{1}$, YANG GUO ${ }^{1}$, QIUYUE HUANG ${ }^{1,2}$, XIN SHI $^{1,2}$, \\ XIAOBO WANG ${ }^{1,3}$ and YING LIU ${ }^{1}$ \\ Schools of ${ }^{1}$ Basic Medical Sciences and ${ }^{2}$ Biomedical Engineering, Hubei University of Medicine, Shiyan, Hubei 442000; \\ ${ }^{3}$ Translational Medicine Center, Suizhou Central Hospital, Hubei University of Medicine, Suizhou, Hubei 441300, P.R. China
}

Received September 10, 2015; Accepted April 3, 2017

DOI: $10.3892 / \mathrm{ol} .2017 .6421$

\begin{abstract}
Gastric cancer is the third most frequent cause of cancer-associated mortality and almost all patients who respond initially to cisplatin (DDP) later develop drug resistance, indicating multi-drug resistance (MDR) is an essential aspect of the failure of treatment. The natural diterpenoid component Oridonin (Ori) has exhibited efficient inhibition in several types of human cancer. However, the effect and potential mechanism of Ori-reversed MDR in human gastric cancer has not been fully elucidated. In the present study, it was found that Ori significantly suppressed DDP-resistant human SGC7901/DDP cell proliferation, growth and colony formation, causing increased caspase-dependent apoptosis, decreased expression of P-glycoprotein (P-gp), encoded by the MDR gene, multi-drug resistance-associated protein (MRP1), and cyclin D1. SGC7901/DDP cells were cultured with different groups of drugs (Ori, DDP alone, or the combination of Ori and DDP). The drug sensitivity, cell apoptosis and effects on MDR were detected by MTT assay and western blot analysis. The results revealed that Ori is able to reverse the DDP resistance and has a clear synergistic effect with DDP in SGC7901/DDP cells by decreasing the levels of P-gp, MRP1, cyclin D1 and cancerous inhibitor of protein phosphatase 2A. Thus, Ori may be a novel effective candidate to treat DDP-resistant human gastric cancer cells.
\end{abstract}

Correspondence to: Dr Ying Liu, School of Basic Medical Sciences, Hubei University of Medicine, 30 South Renmin Road, Shiyan, Hubei 442000, P.R. China

E-mail: ying_liu1002@163.com

Dr Xiaobo Wang, Translational Medicine Center, Suizhou Central Hospital, Hubei University of Medicine, 60 Longmen Street, Suizhou, Hubei 441300, P.R. China

E-mail: wangxiaobo78@126.com

*Contributed equally

Key words: oridonin, gastric cancer, multi-drug resistance, apoptosis

\section{Introduction}

Gastric cancer is one of the most common cancers in Eastern Asia, including China, Japan and South Korea, and Eastern Europe (1). The incidence and mortality of gastric cancer have declined markedly over the past half-century in the majority of developed countries, but it remains the second most common cause of cancer-associated mortality in the world. An estimated 28,000 incident cases $(17,750$ in males and 10,250 in females) of gastric cancer will be diagnosed in 2017, and 10,960 mortalities (6,720 in males and 4,240 in females) are estimated to occur from the disease (2). In China, approximately two-thirds of patients develop advanced or metastatic disease, and $>50 \%$ have recurrent disease following curative surgery (3). Systematic chemotherapy plays a critical role in the treatment of gastric cancer. Cisplatin (DDP) has been commonly used in the treatment of gastric cancer (4). Despite an initial response to surgical debulking and front-line platinum chemotherapy, the majority of tumors eventually develop a drug resistant relapse selected during the course of therapy. The reasons for drug resistance are complicated and several previous studies have aimed to explore the question (1). The development of multidrug resistance (MDR) to cancer chemotherapy is a major obstacle to the effective treatment of advanced gastric cancer (5). Additionally, the mechanism of MDR remains obscure. Mechanisms including increased expression of P-glycoprotein (P-gp) and MDR-associated protein (MRP), cell cycle arrest, increased DNA damage repair and resistance of tumor cells to apoptosis may account for MDR (6). Restoring DDP sensitivity by reversing MDR would be an effective method of treatment.

Oridonin (Ori; Fig. 1A), a bitter tetracyclin diterpenoid compound, may be isolated from Rabdosia rubescens, Isodon japonicus Hara and Isodon trichocarpus, which are widely distributed in China, Japan and Korea (7). Studies show that Ori exhibits potent anticancer activity against a wide range of cancer cell types, including those from prostate, breast, pancreatic and non-small cell lung cancer, leukemia, glioblastoma multiforme and human melanoma cells (8-13). However, to the best of our knowledge, no study has shown the effect and the potential mechanism of Ori reversing MDR of human gastric cancer. Therefore, in the present study, the antitumor effects 
and possible mechanisms of Ori on the DDP-resistant human gastric cancer SGC7901/DDP cell line were investigated.

\section{Materials and methods}

Reagents. Ori with a purity of up to $98 \%$ was purchased from Shanghai YuanYe BioTechnology Co., Ltd. (Shanghai, China). Ori was dissolved in dimethyl sulfoxide (Sigma-Aldrich; Merck KGaA, Darmstadt, Germany) at a stock solution of $100 \mathrm{mM}$ and stored at $-20^{\circ} \mathrm{C}$. DDP was purchased from Sigma-Aldrich (Merck KGaA).

Cell culture. Human cisplatin-resistant gastric cancer cell line SGC7901/DDP and human gastric cancer cell line SGC7901 were purchased from American Type Culture Collection (Manassas, VA, USA). SGC7901/DDP cells were grown in RPMI-1640 (Gibco; Thermo Fisher Scientific, Inc., Waltham, MA, USA) with fetal bovine serum (FBS; Hyclone; GE Healthcare, Logan, UT, USA), $500 \mathrm{ng} / \mathrm{ml}$ DDP, penicillin $(50 \mathrm{U} / \mathrm{ml})$, and streptomycin $(50 \mathrm{U} / \mathrm{ml})$, and incubated in a humidified atmosphere with $\mathrm{CO}_{2}$ at $37^{\circ} \mathrm{C}$. SGC7901 cells were grown in RPMI-1640 with FBS and penicillin $(50 \mathrm{U} / \mathrm{ml})$, and streptomycin $(50 \mathrm{U} / \mathrm{ml})$ and incubated in a humidified atmosphere with $\mathrm{CO}_{2}$ at $37^{\circ} \mathrm{C}$.

Cytotoxic assay and cell viability. Cells were seeded onto a 96-well plate and pre-cultured for $24 \mathrm{~h}$ at $37^{\circ} \mathrm{C}$, then treated with Ori $(5-70 \mu \mathrm{M})$ for $24 \mathrm{~h}$. Cell cytotoxicity was determined by MTT assay (Sigma-Aldrich; Merck KGaA). The absorbance was measured at $490 \mathrm{~nm}$ using the Automated microplate reader (BioTek Instruments, Inc., Winooski, VT, USA), and the inhibition rate was calculated as follows: Inhibition rate $(\%)=\left(\right.$ average $\mathrm{A}_{490}$ of the control group-average $\mathrm{A}_{490}$ of the experimental group)/(average $\mathrm{A}_{490}$ of the control group-average $\mathrm{A}_{490}$ of the blank group) x100. Cell viability was estimated by Trypan blue dye exclusion as previously described (14). The reversion fold calculation formula was used to find the $\mathrm{IC}_{50}$ of DDP on SGC7901/DDP cells/ $\mathrm{IC}_{50}$ of DDP (with $\mathrm{CuB}$ ) on SGC7901/DDP cells. The resistance index (RI) calculation formula was calculated as follows: $\mathrm{RI}=\mathrm{IC}_{50}$ of resistant cells/ $/ \mathrm{IC}_{50}$ of sensitive cells.

Soft-agar colony formation assay. Cells were suspended in $1 \mathrm{ml}$ of RPMI-1640 containing $0.3 \%$ low-melting-point agarose (Amresco, Solon, OH, USA) and 10\% FBS, and plated on a bottom layer containing $0.6 \%$ agarose and $10 \%$ FBS in 6 -well plate in triplicate. After 2 weeks, the plates were stained with $0.2 \%$ gentian violet (Sigma-Aldrich; Merck KGaA) and the colonies were counted under light microscopy (IX70; Olympus Corporation, Tokyo, Japan) (15).

Apoptosis determination by DAPI staining. Approximately $2 \times 10^{5}$ cells/well were added to a 12 -well plate and treated with Ori $(0,20,30,40 \mu \mathrm{M})$ for $24 \mathrm{~h}$. Cells in each treatment and control were then stained by DAPI, examined under fluorescence microscopy and images were captured, as previously described (16).

Western blot analysis. Cell pellets were lysed in radioimmunoprecipitation assay buffer containing $50 \mathrm{mM}$ Tris
(pH 8.0), $150 \mathrm{mM} \mathrm{NaCl}, 0.1 \%$ SDS, $0.5 \%$ deoxycholate, $1 \%$ NP-40, $1 \mathrm{mM}$ DTT, $1 \mathrm{mM} \mathrm{NaF}, 1 \mathrm{mM}$ sodium vanadate and $1 \mathrm{mM}$ PMSF (Sigma-Aldrich; Merck KGaA), and $1 \%$ protease inhibitor cocktail (Merck KGaA). Protein extracts were quantitated and loaded onto $8-12 \%$ sodium dodecyl sulfate polyacrylamide gel, electrophoresed, and transferred to a polyvinylidene difluoride membrane (Merck KGaA). The membrane was incubated overnight at $4^{\circ} \mathrm{C}$ with primary antibody, washed, and then incubated with goat anti-rabbit or anti-mouse immunoglobulin $\mathrm{G}$ (H\&L) horseradish peroxidase (HRP)-conjugated washed, and incubated with HRP-conjugated secondary antibody (1:10,000 dilution; catalog no. E030120-01 and E030110-01; EarthOx, LLC, San Francisco, CA, USA) for $1.5 \mathrm{~h}$ at room temperature for $1.5 \mathrm{~h}$. Detection was performed by using a SuperSignal ${ }^{\circledR}$ West Pico Trial kit (catalog no. QA210131; Pierce Biotechnology, Inc., Rockford, IL, USA). The antibodies used were as follows: Anti-MRP1 (1:500 dilution; catalog no. sc-13960), anti-cyclin D1 (1:500 dilution; catalog no. sc-2044), anti-CIP2A (1:500 dilution; catalog no. sc-80662), anti-Akt (1:500 dilution; catalog no. sc-8312) and anti-phosphorylated (p) Akt (Ser473; 1:500 dilution; catalog no. sc-7985; Santa Cruz Biotechnology, Inc., Dallas, TX, USA); anti-caspase-3 (casp-3; 1:1,000 dilution; catalog no. 9662), anti-PARP (1:1,000 dilution; catalog no. 9542), anti-catalytic subunit of PP2A (PP2Ac; 1:1,000 dilution; catalog no. 2038), anti-extracellular signal-regulated kinase (ERK) 1/2 (1:1,000 dilution; catalog no. 9102) and anti-pERK1/2 (Thr202/Tyr204; 1:1,000 dilution; catalog no. 9101) (Cell Signaling Technology, Inc.); anti-P-gp (1:2,000 dilution; catalog no. ab170904) (Abcam, Cambridge, UK); and anti-GAPDH (1:5,000 dilution; catalog no. M20006; Abmart, Shanghai, China).

Statistical analysis. All experiments were repeated at least three times and the data were presented as the mean \pm standard deviation unless stated otherwise. Differences between data groups were evaluated for significance using Student's t-test for unpaired data or one-way analysis of variance and Bonferroni post hoc test. $\mathrm{P}<0.05$ was considered to indicate a statistically significant difference.

\section{Results}

Effects of Ori on SGC7901 and SGC7901/DDP gastric cancer cells. SGC7901 and SGC7901/DDP cells were seeded onto 96-well plates for $24 \mathrm{~h}$ and then treated with different concentrations of Ori (Fig. 1B and C). After $24 \mathrm{~h}$, the cell viability was evaluated by the MTT assay and absorbance at $490 \mathrm{~nm}$ was measured. It was found that Ori had moderate cytotoxicity to SGC7901 and SGC7901/DDP cells with a half-maximal inhibitory concentration $\left(\mathrm{IC}_{50}\right)$ of 13.84 and $36.35 \mu \mathrm{M}$ (Table I). By Trypan blue exclusion assay, it was found that Ori rapidly reduced the growth of SGC7901/DDP cells (Fig. 1D). The effect of Ori on cell colony formation activity was investigated, and the results showed that Ori significantly inhibited the clonogenic ability of SGC7901/DDP (Fig. 1E). These results suggested that Ori inhibited the anchorage-dependent (cell proliferation) and anchorage-independent (colony formation) growth of SGC7901/DDP cells. 
Table I. $\mathrm{IC}_{50}$ of Ori in gastric cancer cell lines.

\begin{tabular}{lc}
\hline Cell line & $\mathrm{IC}_{50}, \mu \mathrm{M}$ \\
\hline SGC7901 & $13.84 \pm 2.85$ \\
SGC7901/DDP & $36.35 \pm 5.29$
\end{tabular}

Data are shown as the mean \pm standard deviation of quadruplicate determination. $\mathrm{IC}_{50}$, half maximal inhibitory concentration.

Ori induces apoptosis in SGC7901/DDP cells. As aforementioned, the mechanism underlying the inhibition effect of Ori on SGC7901/DDP cells was investigated. The cell death is reminiscent of the phenomena induced by apoptosis. Subsequently, whether Ori induces apoptosis of the SGC7901/DDP cells was assessed. Firstly, the morphological changes of the nucleus were investigated by DAPI staining. As shown in Fig. 2A, nuclear condensation and fragmentation was identified following Ori treatment, which are typical changes in cell apoptosis. Furthermore, western blot analysis was used to detect the activation of the casp-3 effector caspase and its substrate, PARP (Fig. 2B). Ori was demonstrated to induce a significant dose-dependent decrease in pro-casp-3 and the cleavage of PARP, in SGC7901/DDP cells, indicating that Ori induced caspase-dependent apoptosis.

Ori reverses the resistance of SGC7901/DDP cells to DDP. The MTT assay revealed a significant difference between the growth-inhibiting effect of DDP on normal SGC7901 cells and on DDP resistant SGC7901/DDP cells (data not shown). The $\mathrm{IC}_{50}$ for the SGC7901 cells was $14.30 \mu \mathrm{M}$, compared with the $\mathrm{IC}_{50}$ of $34.71 \mu \mathrm{M}$ for the SGC7901/DDP cells. However, the DDP $\mathrm{IC}_{50}$ for SGC7901/DDP cells was $27.87 \mu \mathrm{M}$ subsequent to treatment with $10 \mu \mathrm{M}$ Ori treatment and $14.29 \mu \mathrm{M}$ subsequent to treatment with $20 \mu \mathrm{M}$ Ori (Table II). The resistance index (RI) was 2.43 in the SGC7901/DDP parent group, 1.95 in the SGC7901/DDP Ori $10 \mu \mathrm{M}$ group and 1.00 in the SGC7901/DDP Ori $20 \mu \mathrm{M}$ group. The RI calculation formula was calculated as follows: $\mathrm{RI}=\mathrm{IC}_{50}$ of resistant cells $/ \mathrm{IC}_{50}$ of sensitive cells. Following the treatment with 10 and $20 \mu \mathrm{M}$ Ori, the $\mathrm{IC}_{50}$ of DDP in the SGC7901/DDP cells was reduced from $34.71 \mu \mathrm{M}$ to 27.87 and $14.29 \mu \mathrm{M}$, by 1.25 -fold and 2.43 -fold, respectively.

Ori affected the expression of P-gp, MRPI and cyclin D1. To investigate the mechanism by which Ori induces reversing MDR of SGC7901/DDP cells, the expression levels of P-gp, MRP1 and cell cycle protein cyclin D1 were detected by western blot analysis (Fig. 3). The results indicated that P-gp, MRP1 and cyclin D1 expression of SGC7901/DDP cells were downregulated by treatment with increasing concentrations of Ori. The decreased expression of P-gp and MRP1 in SGC7901/DDP cells may contribute to the reversal of chemoresistance.

Ori inhibited Akt signaling pathway-associated molecules. MDR occurs due to the positive feedback signaling loops generating cancer extreme robustness. Akt is a cancer
A
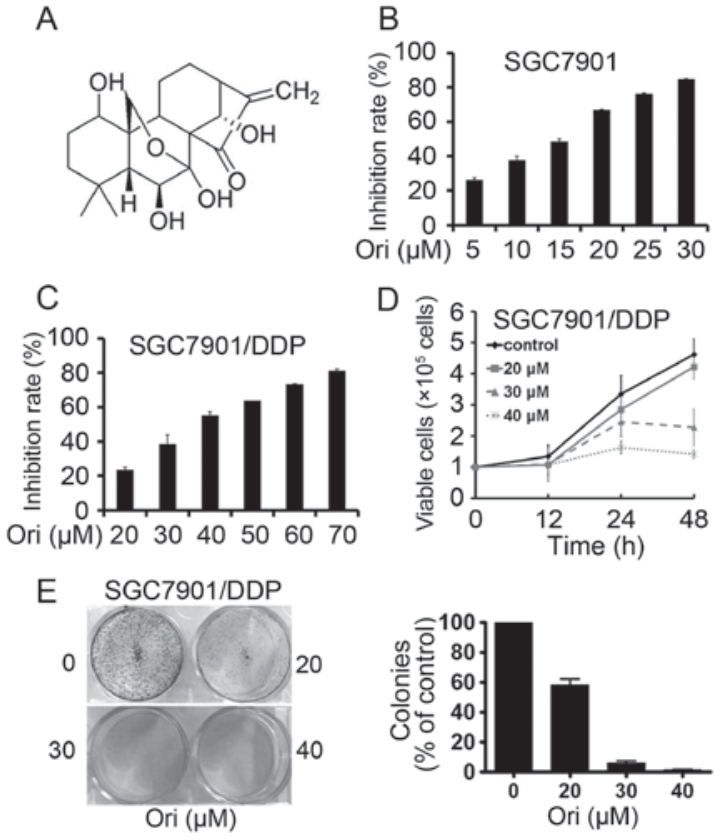

Figure 1. Inhibitory effects of Ori on gastric cancer cells. (A) Chemical structure of Ori. (B and C) The inhibitory effects of Ori on SGC7901 and SGC7901/DDP cells, as analyzed by MTT assay. (D) Inhibitory effects of Ori on cell viability of SGC7901/DDP cells assayed by trypan blue exclusion assay. (E) The colony formation assays of SGC7901/DDP cells treated with Ori at indicated concentration. Ori, oridonin.

MDR locus. Targeting that locus by oxidant/antioxidant balance modulation, positive feedback loops are converted into negative feedback loops, leading to disappearance of MDR (17). The present study examined the effect of Ori on phosphorylated (p)Akt in SGC7901/DDP cells. It was shown that treatment with Ori resulted in downregulation of $\mathrm{pAkt}$, but not phosphorylated (p)ERK1/2 (Fig. 4). PP2A, one of the main serine-threonine phosphatases, has a critical role in the regulation of cell-cycle progression, survival and differentiation by negatively regulating the PI3K/Akt pathway, and dephosphorylating and inactivating mitogen-activated protein kinase kinase 1 and ERK family kinases (18). The present study tested the effects of Ori on PP2Ac (catalytic subunit), and found that Ori upregulated PP2Ac and downregulated cancerous inhibitor of protein phosphatase 2A (CIP2A), a critical oncoprotein in several types of cancer (19), at protein level (Fig. 4). Thus, the present study concluded that the inhibitory effect of Ori on the MDR of SGC7901/DDP cells may be partially due to the regulation of the CIP2A/PP2A/Akt signal cascade.

Ori exerts synergistic effect combining with DDP in SGC7901/DDP cells. The low toxicity (10 and $20 \mu \mathrm{M})$ doses were selected for additional experiments to detect whether Ori can reverse the resistance of SGC7901/DDP cells to low doses of DDP (10 and $20 \mu \mathrm{M})$ (Fig. 5A). Ori in combination with DDP had a greater effect on the SGC-7901/DDP cells compared with DDP or Ori alone $(\mathrm{P}<0.05)$. The combination index (CI) value was also analyzed using CalcuSyn software (version 2.1; Biosoft, Cambridge, UK) and found that the CI values were $<1$ (Table III), which indicated that Ori and DDP played a synergistic effect in SGC7901/DDP cells. Western 

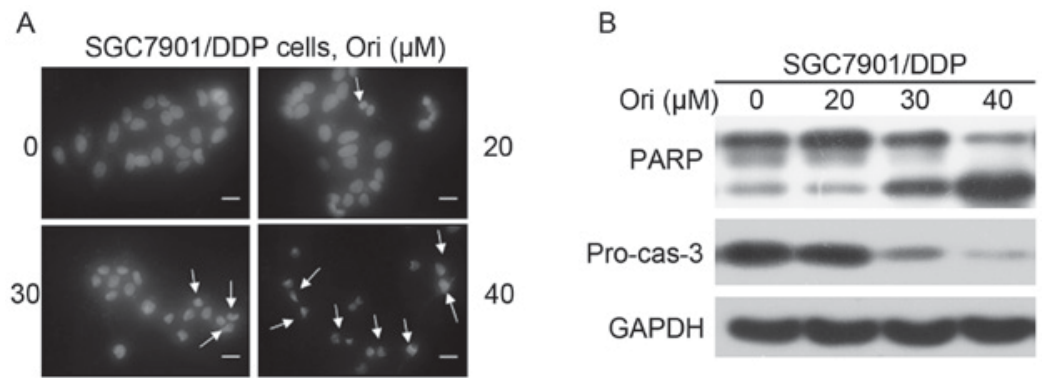

Figure 2. The effects of Ori on apoptosis. (A) SGC7901/DDP cells were incubated with various doses of Ori for $24 \mathrm{~h}$. Cells were examined by DAPI staining. Scale bar=200 $\mu \mathrm{m}$ (B) SGC7901/DDP cells were treated with increasing concentrations of Ori for $24 \mathrm{~h}$. Western blot was conducted using antibodies indicated. Ori, oridonin; PARP, poly (ADP-ribose) polymerase; Pro-cas-3, pro-caspase-3.

Table II. Reversing effect of Ori on SGC7901/DDP cells.

\begin{tabular}{ccccc}
\hline Treatments & $\begin{array}{c}\text { Inhibition } \\
\text { rate, } \%\end{array}$ & $\begin{array}{c}\mathrm{IC}_{50}, \\
\mu \mathrm{M}\end{array}$ & $\begin{array}{c}\text { Resistance } \\
\text { index }\end{array}$ & $\begin{array}{c}\text { Reversion } \\
\text { fold }\end{array}$ \\
\hline $0 \mu \mathrm{M}$ Ori & & 34.71 & 2.43 & 1 \\
$20 \mu \mathrm{M}$ DDP & $29.35 \pm 2.15$ & & & \\
$30 \mu \mathrm{M}$ DDP & $43.92 \pm 3.80$ & & & \\
$40 \mu \mathrm{M}$ DDP & $62.89 \pm 1.53$ & & & \\
$50 \mu \mathrm{M}$ DDP & $62.89 \pm 2.69$ & & & \\
$60 \mu \mathrm{M}$ DDP & $67.40 \pm 5.27$ & & & \\
$70 \mu \mathrm{M}$ DDP & $68.88 \pm 4.04$ & & & \\
$10 \mu \mathrm{M}$ Ori & & 27.87 & 1.95 & \\
$20 \mu \mathrm{M}$ DDP & $32.55 \pm 4.79$ & & & \\
$30 \mu \mathrm{M}$ DDP & $65.83 \pm 2.15$ & & & \\
$40 \mu \mathrm{M}$ DDP & $68.83 \pm 4.36$ & & & \\
$50 \mu \mathrm{M}$ DDP & $70.69 \pm 5.01$ & & & \\
$60 \mu \mathrm{M}$ DDP & $78.05 \pm 3.27$ & & & \\
$70 \mu \mathrm{M}$ DDP & $85.49 \pm 4.62$ & & & \\
$20 \mu \mathrm{M}$ Ori & & 14.29 & 1 & \\
$20 \mu \mathrm{M}$ DDP & $67.50 \pm 2.51$ & & & \\
$30 \mu \mathrm{M}$ DDP & $68.67 \pm 4.01$ & & & \\
$40 \mu \mathrm{M}$ DDP & $72.81 \pm 1.92$ & & & \\
$50 \mu \mathrm{M}$ DDP & $77.89 \pm 3.37$ & & & \\
$60 \mu \mathrm{M}$ DDP & $88.17 \pm 2.95$ & & \\
$70 \mu \mathrm{M}$ DDP & $95.28 \pm 3.52$ & & \\
\hline
\end{tabular}

The DDP IC S0 $_{50}$ of 77901 cell was $14.30 \mu \mathrm{M}$. Data are presented as the mean \pm standard deviation $(n=4)$. DDP, cisplatin; Ori oridonin; $\mathrm{IC}_{50}$, half maximal inhibitory concentration.

blot analysis further confirmed the synergistic effect and found that Ori and DDP combination resulted in elevated levels of casp-3 activation, cleavage of PARP, and decreased P-gp, MRP1, cyclin D1 and CIP2A expression (Fig. 5B).

\section{Discussion}

Gastric cancer is a major type of cancer worldwide and its incidence rate is among the three most frequent neoplasms in China (1). Gastric cancer is also the third most frequent cause of cancer-associated mortality following lung and liver cancer

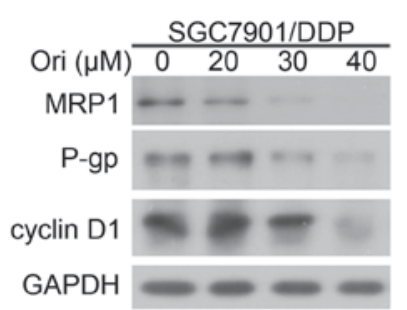

Figure 3. The effects of Ori on multidrug resistance proteins. SGC7901/DDP cells were treated with increasing concentrations of Ori for $24 \mathrm{~h}$. Western blot analysis was conducted using the indicated antibodies. Ori, oridonin; MRP1, multi-drug resistance-associated protein; P-gp, P-glycoprotein.

in males, and following breast and lung cancer in females $(1,2)$. To improve the outcome of gastric cancer, different treatment strategies have been evaluated, including chemotherapy, radiotherapy and extended resection (20). Chemotherapy is the most common treatment of choice for gastric cancer, but its application is limited due to drug resistance. DDP is a platinum chemotherapeutic agent that is widely used in several malignancies and is beneficial in certain patients, but treatment with DDP may cause DDP resistance (21). Therefore, identification and development of novel drugs that can overcome the DDP resistance is required to prolong the overall survival time of gastric cancer patients. To overcome such resistance, exploring novel compounds is urgent. Compounds from natural sources constitute an indispensable candidate drug library for pharmacotherapy. In the present study, the efficacy of the combination of Ori, a bitter tetracyclin diterpenoid compound isolated from traditional medicinal herbs (8), and DDP on the gastric cancer SGC7901/DDP cell line was investigated.

Inhibition of cell proliferation is an efficient strategy in cancer therapy. In this present study, Ori was shown to inhibit the proliferation (Fig. 1C), cell viability (Fig. 1D) and soft-agar colony formation (Fig. 1E) of the gastric cancer SGC7901/DDP cell line.

Evading apoptosis is one of the hallmarks of drug resistance, and targeting apoptosis has become a cancer therapeutic strategy (22). The present study found that the change in cellular morphology and nucleus condensation, which were typical characters of apoptosis (Fig. 2A). Therefore, Ori may have the ability to induce cell apoptosis. The extrinsic and intrinsic apoptotic pathways that ultimately lead to activation of effector caspases (casp-3, -2 and -7) have been characterized $(23,24)$. The decrease in pro-casp- 3 expression and the 
Table III. Ori and DDP CI values.

\begin{tabular}{lc}
\hline Treatment & CI \\
\hline $10 \mu \mathrm{M}$ Ori $+10 \mu \mathrm{M}$ DDP & 0.17 \\
$10 \mu \mathrm{M}$ Ori $+20 \mu \mathrm{M}$ DDP & 0.20 \\
$20 \mu \mathrm{M}$ Ori $+10 \mu \mathrm{M}$ DDP & 0.05 \\
$20 \mu \mathrm{M}$ Ori $+20 \mu \mathrm{M}$ DDP & 0.07 \\
\hline
\end{tabular}

Ori, oridonin; DDP, cisplatin; CI, combination index.

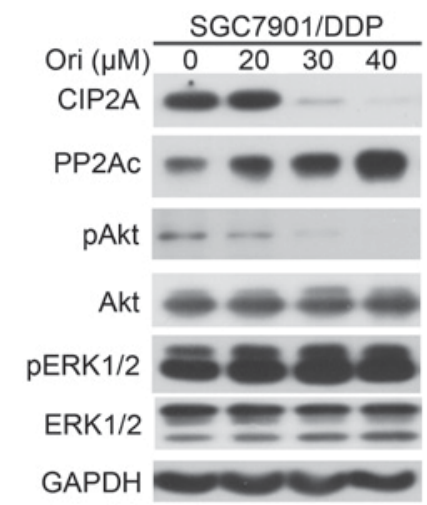

Figure 4. The effects of Ori on Akt signaling associated molecules. SGC7901/DDP cells were treated with increasing concentrations of Ori for $24 \mathrm{~h}$. Western blot analysis was conducted using the indicated antibodies. Ori, oridonin; CIP2A, cancerous inhibitor of protein phosphatase 2A; PP2Ac, catalytic subunit of protein phosphatase 2A; pAkt, phosphorylated Akt; ERK1/2, extracellular signal-regulated kinase 1/2; pERK1/2, phosphorylated ERK1/2.
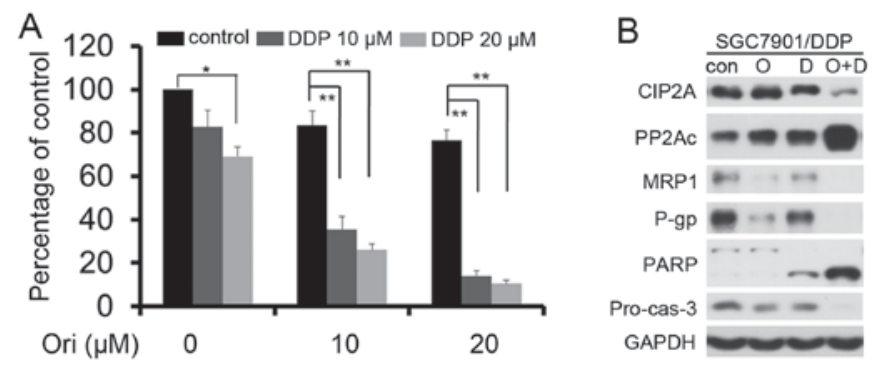

Figure 5. Ori exerts synergistic effects in combination with DDP (A) SGC7901/DDP cells were treated for $24 \mathrm{~h}$ with Ori and/or DDP, and then assessed by MTT assay. "P $<0.05$; ${ }^{* *} \mathrm{P}<0.01$. (B) SGC7901/DDP cells were treated with Ori $(\mathrm{O} ; 20 \mu \mathrm{M})$, DDP $(\mathrm{D} ; 20 \mu \mathrm{M})$ alone or together $(\mathrm{O}+\mathrm{D})$ for $24 \mathrm{~h}$. The treated cells were collected, lysed and assessed by western blot analysis using the indicated antibodies. DDP, cisplatin; Ori oridonin; CIP2A, cancerous inhibitor of protein phosphatase $2 \mathrm{~A} ; \mathrm{PP} 2 \mathrm{Ac}$, catalytic subunit of protein phosphatase $2 \mathrm{~A}$; MRP1, multi-drug resistance-associated protein; P-gp, P-glycoprotein; PARP, poly (ADP-ribose) polymerase; Pro-cas-3, pro-caspase-3.

proteolysis of PARP (Fig. 2B) indicate that casp-3 is activated. Thus, Ori may trigger apoptosis by activating the casppasedependent apoptosis pathway.

A number of experimental strategies to overcome DDP resistance act at the preclinical or clinical levels, including the introduction of pro-apoptotic genes and the inhibition of antioxidants that protect against oxidative stress and prevent damage to cells $(25,26)$. Of particular significance are the combinations of chemotherapy drug treatments with other drugs, radiation and the emerging gene therapy regimens. The effect of the combination of Ori and DDP on the SGC7901/DDP cells was investigated, and it was identified that Ori in combination with DDP had an improved effect compared with DDP or Ori alone. Following treatment with Ori $(10$ and $20 \mu \mathrm{M})$, the $\mathrm{IC}_{50}$ of DDP to the SGC7901/DDP cells was significantly reduced from $34.71 \mu \mathrm{M}$ to 27.87 and $14.29 \mu \mathrm{M}$ by 1.25 -fold and 2.43 -fold, respectively (Table II). The RI was 2.43 in the SGC7901/DDP parent group, 1.95 in the SGC7901/DDP Ori $10 \mu \mathrm{M}$ group and 1.00 in the SGC7901/DDP Ori $20 \mu \mathrm{M}$ group. Furthermore, the present study explored the mechanisms of Ori in reversing DDP resistance.

Drug resistance is largely mediated through overexpression of MDR, MRP, drug resistance protein and proteasome subunits, increases in antioxidant defenses, and TOP2 activity; these results have been widely verified (27-29). In addition, suppression of cyclin D1 levels has been shown to potentiate the response of human pancreatic cancer cells to DDP (30). The present study identified that the treatment with Ori was able to reverse the MDR of the SGC7901/DDP cells via the downregulation of P-gp, MRP1 and cyclin D1 (Fig. 3).

To assess whether Akt and CIP2A are the targets of Ori, their activation and expression in SGC7901/DDP cells treated with Ori was measured. Akt phosphorylation and CIP2A expression were inhibited by treatment with Ori (Fig. 4). This suggests that Ori reverses MDR, at least in part, through suppression of the CIP2A/PP2A/Akt signaling cascade.

The low toxicity (10 and $20 \mu \mathrm{M})$ doses of Ori and DDP were combined to investigate a possible synergistic effect, and the results showed that Ori could significantly elevate DDP anti-tumor efficiency, and the CI was $<1$, as calculated by CalcuSyn software (version 2.1). Additionally, the expression of full-length casp-3 was reduced and PARP was cleaved. Additionally, the MDR genes P-gp and MRP1, and CIP2A were also significantly inhibited by combined treatment with Ori and DDP. Cisplatin, which reacts with DNA and forms DNA adducts, is the main drug for treatment of gastric cancer (26). Notably, the two drugs, which have different mechanisms, synergistically inhibited cell growth. The combination of these drugs may have clinic potential in cisplatin-resistant human gastric cancer treatment.

In conclusion, the present results have confirmed that Ori combination with DDP could overcome chemoresistance of SGC7901/DDP cells. One possible mechanism is that Ori induced apoptosis, modulated drug resistance and cell cycle proteins, P-gp, MRP1 and cyclin D1. The inhibition of the CIP2A/PP2A/Akt signal cascade pathway is a key role in the process of Ori plays its function. Ori may be a promising new drug due to its ability to reverse MDR in gastric cancer.

\section{Acknowledgements}

The present study was supported by grants from the Natural Science Foundation of Hubei Province (grant no. 2016CFB528); the Foundation of Health and Family planning Commission of Hubei Province (grant no. WJ2017F065); the Foundation of Hubei University of Medicine (grant no. FDFR201605); the 
Foundation for Innovative Research Team of Hubei University of Medicine (grant no. 2014CXX05) and the Key Discipline Project of Hubei University of Medicine.

\section{References}

1. Niccolai E, Taddei A, Prisco D and Amedei A: Gastric cancer and the epoch of immunotherapy approaches. World J Gastroenterol 21: 5778-5793, 2015.

2. Siegel RL, Miller KD and Jemal A: Cancer statistics, 2015. CA Cancer J Clin 65: 5-29, 2015

3. Yang X, Cai H, Liang Y, Chen L, Wang X, Si R, Qu K, Jiang Z, $\mathrm{Ma} \mathrm{B}$, Miao C, et al: Inhibition of c-Myc by let-7b mimic reverses mutidrug resistance in gastric cancer cells. Oncol Rep 33: 1723-1730, 2015.

4. Ni P, Xu W, Zhang Y, Chen Q, Li A, Wang S, Xu S and Zhou J: TXNL1 induces apoptosis in cisplatin resistant human gastric cancer cell lines. Current Cancer Drug Targets 14: 850-859, 2015.

5. Fan K, Fan D, Cheng LF and Li C: Expression of multidrug resistance-related markers in gastric cancer. Anticancer Res 20 : 4809-4814, 2000

6. Hong L, Piao Y, Han Y, Wang J, Zhang X, Du Y, Cao S, Qiao T, Chen Z and Fan D: Zinc ribbon domain-containing 1 (ZNRD1) mediates multidrug resistance of leukemia cells through regulation of P-glycoprotein and Bcl-2. Mol Cancer Ther 4: 1936-1942, 2005.

7. Owona BA and Schluesener HJ: Molecular insight in the multifunctional effects of oridonin. Drugs R D 15: 233-244, 2015.

8. Zhou GB, Kang H, Wang L, Gao L, Liu P, Xie J, Zhang FX, Weng XQ, Shen ZX, Chen J, et al: Oridonin, a diterpenoid extracted from medicinal herbs, targets AML1-ETO fusion protein and shows potent antitumor activity with low adverse effects on $\mathrm{t}(8 ; 21)$ leukemia in vitro and in vivo. Blood 109 3441-3450, 2007.

9. Xu B, Shen W, Liu X, Zhang T, Ren J, Fan Y and Xu J: Oridonin inhibits BxPC-3 cell growth through cell apoptosis. Acta Biochim Biophys Sin (Shanghai) 47: 164-173, 2015.

10. Li Y, Wang Y, Wang S, Gao Y, Zhang X and Lu C: Oridonin phosphate-induced autophagy effectively enhances cell apoptosis of human breast cancer cells. Med Oncol 32: 365, 2015.

11. Chen RY, Xu B, Chen SF, Chen SS, Zhang T, Ren J and Xu J: Effect of oridonin-mediated hallmark changes on inflammatory pathways in human pancreatic cancer (BxPC-3) cells. World J Gastroenterol 20: 14895-14903, 2014.

12. Wang YY, Lv YF, Lu L and Cai L: Oridonin inhibits mTOR signaling and the growth of lung cancer tumors. Anticancer Drugs 25: 1192-1200, 2014.

13. Zhao Z and Chen Y: Oridonin, a promising antitumor natural product in the chemotherapy of hematological malignancies. Curr Pharm Biotechnol 15: 1083-1092, 2014.

14. Liu Y, Cao W, Zhang B, Liu YQ, Wang ZY, Wu YP, Yu XJ, Zhang XD, Ming PH, Zhou GB and Huang L: The natural compound magnolol inhibits invasion and exhibits potential in human breast cancer therapy. Sci Rep 3: 3098, 2013.

15. Cao W, Liu Y, Zhang R, Zhang B, Wang T, Zhu X, Mei L, Chen $\mathrm{H}$, Zhang $\mathrm{H}$, Ming $\mathrm{P}$ and Huang L: Homoharringtonine induces apoptosis and inhibits STAT3 via IL-6/JAK1/STAT3 signal pathway in Gefitinib-resistant lung cancer cells. Sci Rep 5 8477, 2015.
16. Chou CC, Yang JS, Lu HF, Ip SW, Lo C, Wu CC, Lin JP, Tang NY, Chung JG, Chou MJ, et al: Quercetin-mediated cell cycle arrest and apoptosis involving activation of a caspase cascade through the mitochondrial pathway in human breast cancer MCF-7 cells Arch Pharm Res 33: 1181-1191, 2010.

17. Radisavljevic Z: AKT as locus of cancer multidrug resistance and fragility. J Cell Physiol 228: 671-674, 2013.

18. Liu H, Gu Y, Wang H, Yin J, Zheng G, Zhang Z, Lu M, Wang C and $\mathrm{He} \mathrm{Z}$ : Overexpression of PP2A inhibitor SET oncoprotein is associated with tumor progression and poor prognosis in human non-small cell lung cancer. Oncotarget 6: 14913-14925, 2015.

19. Khanna A and Pimanda JE: Clinical significance of cancerous inhibitor of protein phosphatase $2 \mathrm{~A}$ in human cancers. Int $\mathrm{J}$ Cancer 138: 525-532, 2016.

20. Zhang JL, Liu XZ, Wang PY, Chen GW, Jiang Y, Qiao SK, Zhu J, Wang X, Pan YS and Liu YC: Targeting HCCR expression resensitizes gastric cancer cells to chemotherapy via down-regulating the activation of STAT3. Sci Rep 6: 24196, 2016.

21. Simonian PL, Grillot DA and Nuñez G: Bcl-2 and Bcl-XL can differentially block chemotherapy-induced cell death. Blood 90: 1208-1216, 1997.

22. Hanahan D and Weinberg RA: Hallmarks of cancer: The next generation. Cell 144: 646-674, 2011.

23. Nicholson DW: Caspase structure, proteolytic substrates, and function during apoptotic cell death. Cell Death Differ 6: 1028-1042, 1999 .

24. Johnstone RW, Ruefli AA and Lowe SW: Apoptosis: A link between cancer genetics and chemotherapy. Cell 108: 153-164, 2002.

25. Zhao Z, Wang J, Tang J, Liu X, Zhong Q, Wang F, Hu W, Yuan Z, Nie $\mathrm{C}$ and Wei Y: JNK- and Akt-mediated Puma expression in the apoptosis of cisplatin-resistant ovarian cancer cells. Biochem J 444: 291-301, 2012.

26. Hasegawa M, Ishiguro K, Ando $\mathrm{T}$ and Goto H: Geranylgeranylacetone attenuates cisplatin-induced reductions in cell viability by suppressing the elevation of intracellular p53 content without heat shock protein induction. Nagoya J Med Sci 74: 123-131, 2012.

27. Alcantara LM, Kim J, Moraes CB, Franco CH, Franzoi KD, Lee S, Freitas-Junior LH and Ayong LS: Chemosensitization potential of P-glycoprotein inhibitors in malaria parasites. Exp Parasitol 134: 235-243, 2013.

28. Luo L, Sun YJ, Yang L, Huang S and Wu YJ: Avermectin induces P-glycoprotein expression in S2 cells via the calcium/calmodulin/NF-kappaB pathway. Chem Biol Interact 203: 430-439, 2013.

29. Ying L, Zu-An Z, Qing-Hua L, Qing-Yan K, Lei L, Tao C and Yong-Ping W: RAD001 can reverse drug resistance of SGC7901/DDP cells. Tumour Biol 35: 9171-9177, 2014.

30. Kornmann M, Arber N and Korc M: Inhibition of basal and mitogen-stimulated pancreatic cancer cell growth by cyclin D1 antisense is associated with loss of tumorigenicity and potentiation of cytotoxicity to cisplatinum. J Clin Invest 101: 344-352, 1998. 